\title{
Electrical and optical characteristics of AIAsSb/GaAsSb distributed Bragg reflectors for surface emitting lasers
}

\author{
O. Blum, ${ }^{\text {a) }}$ M. J. Hafich, J. F. Klem, and K. L. Lear \\ Sandia National Laboratories, MS0603, P.O. Box 5800, Albuquerque, New Mexico 87185 \\ S. N. G. Chu \\ AT\&T Bell Laboratories, Murray Hill, New Jersey 07974
}

(Received 24 July 1995; accepted for publication 20 September 1995)

\begin{abstract}
We demonstrate an undoped $20 \frac{1}{2}$ pair AlAsSb/GaAsSb distributed Bragg reflector (DBR) grown lattice matched to an InP substrate by molecular beam epitaxy. Reflectivity measurements indicate a stop band centered at $1.78 \mu \mathrm{m}$ with a maximum reflectivity exceeding $99 \%$. We also measure current-voltage characteristics in a similar $10 \frac{1}{2}$ period $p$-type DBR and find that a current density of $1 \mathrm{kA} / \mathrm{cm}^{2}$ produces a $2.5 \mathrm{~V}$ drop. Hole mobilities and doping concentrations in AlAsSb and GaAsSb are also reported. (c) 1995 American Institute of Physics.
\end{abstract}

Vertical-cavity surface-emitting lasers (VCSELs) have been demonstrated at a variety of wavelengths shorter than 1 $\mu \mathrm{m} .{ }^{1,2}$ They have found many useful applications from short haul optical links to printing. Demonstration of similar devices at wavelengths longer than $1 \mu \mathrm{m}$ has been more problematic. This is because refractive index differences $(\Delta n)$ are relatively small between materials in the InGaAsP system, which is most commonly used in this wavelength range, and the distributed Bragg reflector (DBR) optical performance depends on $\Delta n$ between the mirror layers. One approach to this problem has been wafer bonding of GaAs/AlGaAs DBRs to InP/InGaAsP active regions. In this manner, pulsed 1.3 (Ref. 3) and $1.55 \mu \mathrm{m}$ (Ref. 4) VCSELs have been demonstrated. Progress has also been made using thermally conductive dielectric mirrors. ${ }^{5}$ An alternative all-epitaxial approach consists of using DBRs composed of antimonide compounds, which can be lattice matched to InP and have a high refractive index contrast.

Recently, several groups have demonstrated highly reflective DBRs incorporating various antimonide compounds including: AlPSb/GaPSb on $\mathrm{InP}^{6}{ }^{6} \mathrm{GaAsSb} / \mathrm{AlAsSb}$ on $\mathrm{InP},{ }^{7}$ and $\mathrm{AlGaAsSb} / \mathrm{AlAsSb}$ on $\mathrm{InP}^{8,9}$ All these structures were undoped, however, whereas an electrically injected VCSEL usually employs at least one doped semiconductor mirror. The issues of doping in these materials have not been explored in the context of DBR optical properties and electrical transport. As with VCSELs emitting at shorter wavelengths, the properties of doped DBRs can have a fundamental effect on the VCSEL performance, due to free carrier loss and thermal dissipation in the mirror. These are particularly critical for the $p$-type mirror, because of lower hole mobility and larger effective masses.

In this letter, we report the highest demonstrated reflectivity for an undoped GaAsSb/AlAsSb DBR $(R>99 \%)$, as well as a $p$-type GaAsSb/AlAsSb DBR and its optical and electrical characteristics. Both mirrors were grown by molecular beam epitaxy (MBE) lattice matched to InP with center wavelengths at 1.78 and $1.74 \mu \mathrm{m}$, respectively. The main

${ }^{a)}$ Electronic mail: oblum@sandia.gov advantage of this material system is a large $\Delta n \sim 0.5$, which enables fabrication of DBRs with relatively few mirror pairs and allows higher ultimate reflectivities in the presence of free carrier absorption. Lattice matching to InP also facilitates compatibility with the more mature InGaAs/InGaAsP materials for the active region. Shifting the absorption edge of the high refractive index layer toward shorter wavelengths by using $\mathrm{Al}_{0.1} \mathrm{Ga}_{0.9} \mathrm{As}_{0.518} \mathrm{Sb}_{0.418}$ rather than $\mathrm{GaAs}_{0.51} \mathrm{Sb}_{0.49}$, will enable growth of DBRs with stop bands centered at 1.55 $\mu \mathrm{m}$. We have demonstrated this approach through the use of digital alloys, ${ }^{9}$ but the homogeneous alloy approach may benefit the electronic transport properties of doped DBRs. However, the homogeneous approach requires two Al cells, since Al composition cannot be changed rapidly enough using a single $\mathrm{Al}$ cell without long interrupts. GaAsSb/AlAsSb DBRs are suitable for operation near $1.75 \mu \mathrm{m}$, a wavelength important for environmental monitoring applications, because of overlap with the first overtone of the $\mathrm{C}-\mathrm{H}$ stretch. ${ }^{10}$

The AlAsSb/GaAsSb epitaxial growth is performed by solid source MBE. Both group V elements are used in the tetramer form. An effusion cell controls arsenic overpressure and antimony flux is produced from a low temperature effusion cell. The growth rate for each of the alloys is primarily determined by the $\mathrm{Al}$ or $\mathrm{Ga}$ arrival rate; both $\mathrm{Ga}$ and $\mathrm{Al}$ fluxes are adjusted prior to alloy growth using reflection high energy electron diffraction oscillations during binary growth of GaAs or AlAs on a separate GaAs substrate. Alloy composition is measured using $\mathrm{x}$-ray diffraction and regulated with beam equivalent pressure measurements of the $\mathrm{Sb}_{4}$ flux, while maintaining a fixed overpressure of $\mathrm{As}_{4}$ flux. The $\mathrm{AlAsSb} / \mathrm{GaAsSb} \mathrm{DBR}$ structures are grown with constant fluxes of $\mathrm{As}_{4}$ and $\mathrm{Sb}_{4}$ on Fe-doped (semi-insulating) InP substrates heated to $520{ }^{\circ} \mathrm{C}$. The undoped DBR consists of 20 pairs of $\mathrm{GaAs}_{0.51} \mathrm{Sb}_{0.49} / \mathrm{AlAs}_{0.56} \mathrm{Sb}_{0.44}$ layers, 1200 and 1453 $\AA$ thick, respectively, terminating on the substrate side with an additional $1200 \AA$ thick intrinsic $\mathrm{GaAs}_{0.51} \mathrm{Sb}_{0.49}$ layer. The inset in Fig. 1 illustrates the layer sequence of this structure, which has a the total thickness of $5.4 \mu \mathrm{m}$.

A cross-sectional transmission electron micrograph (TEM) of the DBR sample is shown in Fig. 2. The dark and 


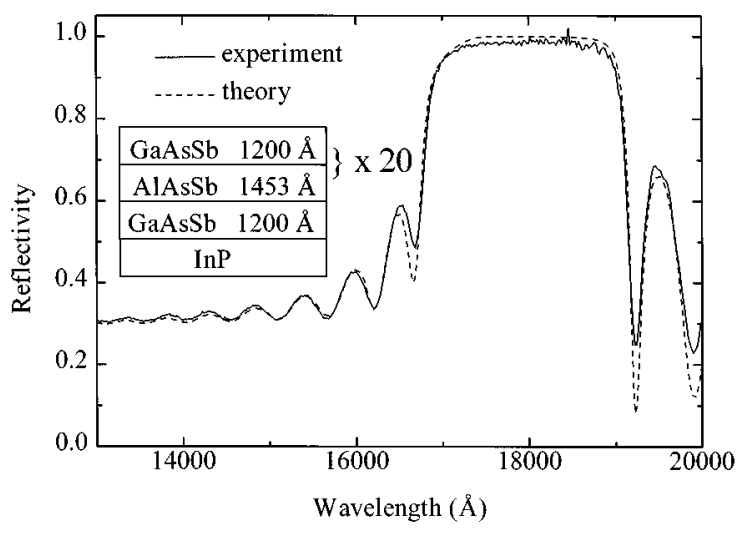

FIG. 1. Experimental reflectivity measurement (shown with a solid line) and theoretical prediction of reflectivity (shown with a dashed line) for the undoped GaAsSb/AlAsSb DBR. Shown in the inset is the schematic representation of the DBR layer structure. The mirror consists of $20 \frac{1}{2}$ pairs of $\mathrm{GaAs}_{0.51} \mathrm{Sb}_{0.49} / \mathrm{AlAs}_{0.56} \mathrm{Sb}_{0.44}$ layers, with approximate thicknesses of 1200 and $1453 \AA$, respectively.

light alternating areas correspond to the GaAsSb and AlAsSb regions respectively. Sharp interfaces between the two materials can be observed. The TEM images show the DBR layers to be very homogeneous, flat and defect-free. X-ray diffraction data taken on the DBR sample indicate that lattice match to the InP substrate is $|\Delta a / a| \leqslant 0.1 \%$. Growth of nearly defect-free material can be credited with an improvement in lattice match relative to our previous efforts. ${ }^{7}$

The reflectivity spectrum of the DBR is shown in Fig. 1. The solid line indicates the experimental reflectivity, while the dashed line curve represents the theoretically predicted reflectivity using adjusted thickness parameters. Details of

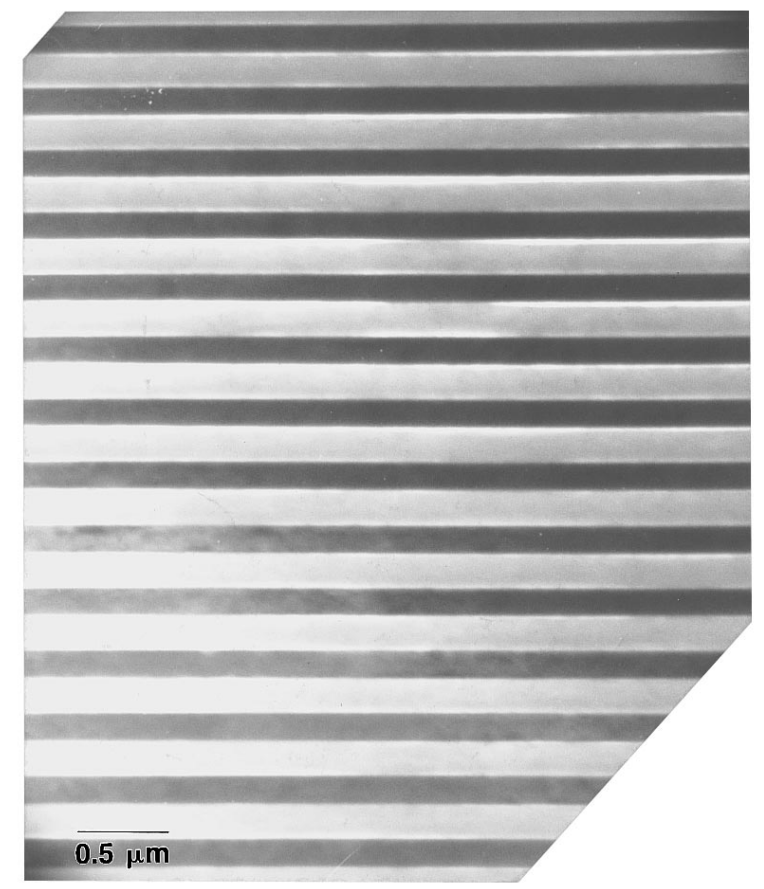

FIG. 2. Cross-sectional transmission electron microscopy image of the DBR. Fourteen mirror pairs of the DBR are visible (dark areas correspond to $\mathrm{GaAsSb}$ and light areas to AlAsSb). reflectivity modeling and optical constants used are described elsewhere. ${ }^{7}$ In order to obtain the best fit, we used $1474 \AA$ for AlAsSb layers and $1234 \AA$ for GaAsSb layers, except for the GaAsSb layer closest to the surface, which was $1184 \AA$. These values are very close to the nominal thicknesses of 1453 and $1200 \AA$, respectively. In addition, the theoretical curve for the $20 \frac{1}{2}$ period DBR yields a stop band centered at $1784 \mathrm{~nm}$ with a maximum reflectivity of $\sim 99.95 \%$ and a full width of $215 \mathrm{~nm}$, while the measured result indicates a $212 \mathrm{~nm}$ wide stop band, centered at 1782 $\mathrm{nm}$ and a maximum reflectivity of $>99 \%$. Clearly, a very good fit is obtained, in terms of agreement with the stop band full width, position and peak value, as well as the positions of the sidebands. The excellent agreement between experimental and theoretical values of the stop band full width is an indication of the accuracy of the refractive index model for the two DBR layers, since its value is determined by the refractive index constant.

The $p$-type DBR was grown using the same conditions as the undoped DBR described previously. The structure consists of $10 \frac{1}{2}$ pairs of $\mathrm{GaAs}_{0.51} \mathrm{Sb}_{0.49} / \mathrm{AlAs}_{0.56} \mathrm{Sb}_{0.44}$ layers, which are 1200 and $1453 \AA$ thick, respectively. A $p$-type, $\mathrm{Zn}$-doped InP substrate was used so that the current-voltage characteristics could be measured. From X-ray diffraction we determined that the lattice mismatch to the InP substrate is $|\Delta a / a| \leqslant 0.2 \%$. The $p$-type mirror layers were doped with Be. Hall measurements on $1 \mu \mathrm{m}$ layers of Be-doped GaAsSb and $1 \mu \mathrm{m}$ layers of Be-doped AlAsSb determined the actual hole densities and mobilities to be $7.6 \times 10^{18} \mathrm{~cm}^{-3}$ and $\mu=35 \mathrm{~cm}^{2} / \mathrm{V} \mathrm{s}$ for GaAsSb, and $4.4 \times 10^{18} \mathrm{~cm}^{-3}$ and $\mu=6.6 \mathrm{~cm}^{2} / \mathrm{V} \mathrm{s}$ for AlAsSb at $300 \mathrm{~K}$. The mobility for $\mathrm{GaAsSb}$ agrees quite well with that reported in the literature, ${ }^{11}$ whereas the value for AlAsSb is believed to be the first reported. The $10 \frac{1}{2}$ period $p$-type DBR was found to have a stop band centered at $1.74 \mu \mathrm{m}$ with a maximum reflectivity of $98.8 \%$ and a full width of $210 \mathrm{~nm}$. The theoretically predicted values were $1.75 \mu \mathrm{m}, 98 \%$ and $199 \mathrm{~nm}$ for the stop band center, maximum reflectivity and full width, respectively. The measured values of maximum reflectivity and full width are slightly larger than those predicted theoretically due to an overestimation of the band edge absorption of GaAsSb layers used in the model. Free carrier absorption does not appear to have a significant effect, but its true impact can only be evaluated from measurements of laser loss, rather than from reflectivity of the DBR alone. Our simulations indicate that for this mirror stack, if we assume a free carrier absorption of $50 \mathrm{~cm}^{-1}$ (a conservative estimate several times larger than that routinely used for GaAs/ AlGaAs DBRs) the decrease in the maximum reflectivity over a mirror with no absorption would be less than $0.2 \%$.

The $p$-type DBRs were patterned into $30 \mu \mathrm{m}$ diameter mesas and dry etched by reactive ion etching (RIE) through the entire DBR. Contacts of $\mathrm{Ti} / \mathrm{Pd} / \mathrm{Au}$ and $\mathrm{Au} / \mathrm{Be}$ were evaporated on mesa tops and sample backside, respectively, and annealed at $350{ }^{\circ} \mathrm{C}$ for $30 \mathrm{~s}$. The details of the resistance measurement are described elsewhere. ${ }^{12}$ The contact resistance was measured to be $\sim 1 \times 10^{-4} \Omega \mathrm{cm}^{2}$. The currentvoltage $(I-V)$ characteristics of the $p$-type stack, including 


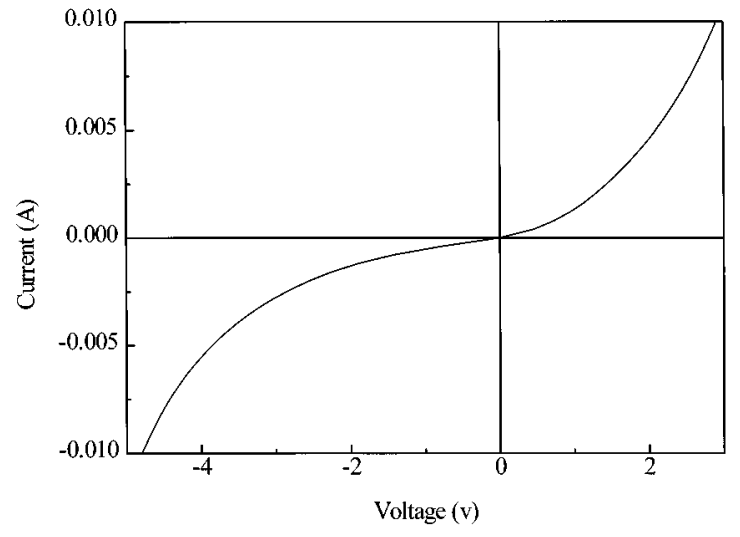

FIG. 3. Current-voltage characteristic of the $p$-type AlAsSb/GaAsSb 10 1/2 period DBR.

negligible contributions from contact and substrate resistance, are shown in Fig. 3. We found that $1 \mathrm{kA} / \mathrm{cm}^{2}$ of current density corresponds to a $\sim 2.5 \mathrm{~V}$ drop in the stack mobilities. Using the mobilities and dopings obtained from Hall measurement we estimate that at this current density $\left(1 \mathrm{kA} / \mathrm{cm}^{2}\right) \sim 35 \mathrm{mV}$ of the voltage drop can be attributed to the bulk resistance of the DBRs, even with the low bulk mobility in the AlAsSb layers. The voltage drop is thus dominated by the band offsets between GaAsSb and AlAsSb. While these are not known experimentally, we have inferred their values from measurements of band offsets between $\mathrm{AlAsSb}$ and InGaAs ${ }^{13}$ and GaAsSb and InGaAs, ${ }^{14}$ under the assumption of transitivity. In this way we find a type I band alignment with a $\Delta E_{c}$ of 1.27 and a $\Delta E_{v}$ of $0.4 \mathrm{eV}$ $\left[E_{g}(\mathrm{AlAsSb})>E_{g}(\mathrm{GaAsSb})\right]$, where $E_{g}(\mathrm{AlAsSb})=2.39$ $\mathrm{eV}^{13}$ and $E_{g}(\mathrm{GaAsSb})=0.72 \mathrm{eV}$. ${ }^{14}$ The use of graded interface schemes such as those in shorter wavelength VCSELs, ${ }^{15}$ has resulted in the improvement of specific resistivity of the DBRs by two orders of magnitude. Thus employing similar techniques in the $\mathrm{AlAsSb} / \mathrm{GaAsSb}$ mirror stacks can be expected to greatly reduce the voltage drop due to band offsets, as well.
In conclusion, we present the highest reflectivity undoped $\mathrm{AlAsSb} / \mathrm{GaAsSb}$ mirror stack demonstrated to date. We also report for the first time the optical and electrical characteristics of a $p$-type AlAsSb/GaAsSb DBR. Our model successfully predicts the optical characteristics of the DBR. These results indicate that the AlGaAsSb material system can be useful for surface normal optoelectronic devices operating in the $1.55-1.75 \mu \mathrm{m}$ wavelength range because it can provide highly reflecting, conductive DBRs lattice matched to $\mathrm{InP} / \mathrm{InGaAs}$ active regions.

We gratefully acknowledge expert help from R. J. Winkelman, J. A. Nevers, and S. Kilcoyne. This work was supported by the U.S. Department of Energy under Contract No. DE-AC04-76-DP00789.

${ }^{1}$ F. Koyama, S. Kinoshita, and K. Iga, Appl. Phys. Lett. 55, 221 (1989).

${ }^{2}$ Y. H. Lee, J. L. Jewell, A. Scherer, S. L. McCall, S. J. Walker, J. P. Harbison, and L. T. Florez, Electron. Lett. 25, 1377 (1989).

${ }^{3}$ J. J. Duley, D. I. Babic, R. P. Mirin, L. Yang, B. I. Miller, R. J. Ram, T. Reynolds, E. L. Hu, and J. E. Bowers, IEEE Trans. Electron. Devices ED-40, 2119 (1993).

${ }^{4}$ D. I. Babic, I. Hsing Tan, R. P. Mirin, J. E. Bowers, and E. L. Hu, Technical Digest of Conference on Laser and Electro-optics, Baltimore, Maryland, May 1995, paper CWB7.

${ }^{5}$ T. Baba, Y. Yogo, K. Suzuki, F. Koyama, and K. Iga, Jpn. J. Appl. Phys. 33, 1905 (1994).

${ }^{6}$ T. Anan, H. Shimomura, and S. Sugou, Electron. Lett. 30, 2138 (1994).

${ }^{7}$ O. Blum, I. J. Fritz, L. R. Dawson, A. J. Howard, T. J. Headley, J. F. Klem, and T. J. Drummond, Appl. Phys. Lett. 66, 329 (1995).

${ }^{8}$ B. Lambert, Y. Toudic, Y. Rouillard, M. Gauneau, M Baudet, F. Alard, I. Valiente, and J. C. Simons, Appl. Phys. Lett. 66, 442 (1995).

${ }^{9}$ O. Blum, I. J. Fritz, L. R. Dawson, and T. J. Drummond, Electron. Lett. 31, 1247 (1995).

${ }^{10}$ B. Stallard, M. J. Garcia, and S.Kaushik (unpublished).

${ }^{11}$ G. J. Sullivan, C. W. Farley, W. J. Ho, R. L. Pierson, M. K. Szwed, M. D. Lind, and R. L. Bernescut, J. Electron. Mater. 21, 1123 (1992).

${ }^{12}$ K. L. Lear and R. P. Schneider, Jr. (unpublished).

${ }^{13}$ T. Inata, S. Muto, Y. Nakata, and T. Fujii, Jpn. J. Appl. Phys. 29, L1382 (1990).

${ }^{14}$ Y. Sugiyama, T. Fujii, Y. Nakata, S. Muto, and E. Miyauchi, J. Cryst. Growth 95, 363 (1989).

${ }^{15}$ K. Tai, L. Yang, Y. H. Wang, and J. D. Wynn, Appl. Phys. Lett. 56, 2496 (1990) 